\section{Major rectal bleeding following transrectal needle prostate biopsy}

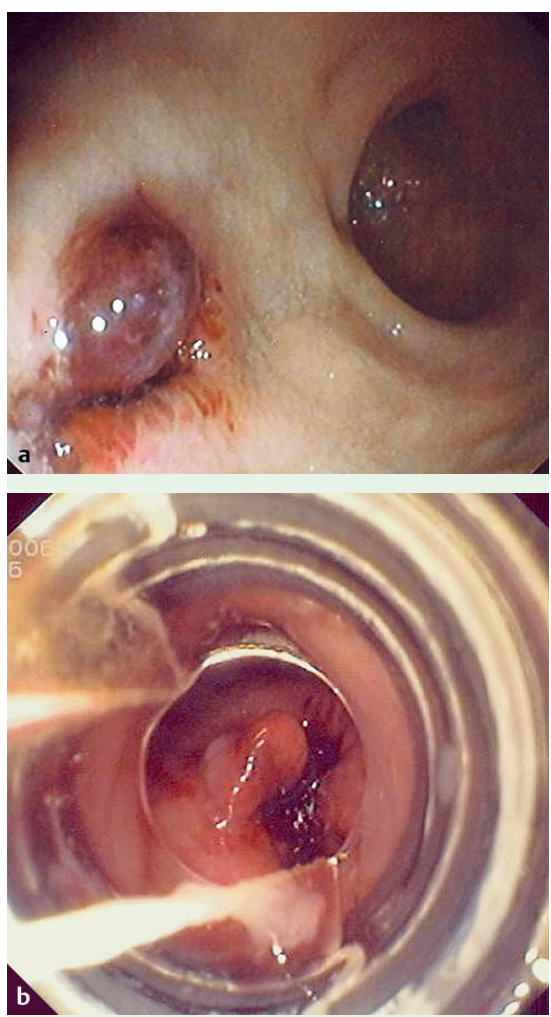

Fig. 1 a Blood vessel visualized on the anterior rectal wall. b Elastic band ligation (EBL) being carried out.

Transrectal ultrasound (TRUS)-guided prostate core biopsy is a very useful diagnostic tool in urological diseases. It constitutes a simple and safe outpatient procedure in the majority of patients, although hemorrhagic complications, ranging from transient hematuria to catastrophic rectal bleeding, may occur [1]. Rectal bleeding affects up to $50 \%$ of patients, although only $1 \%$ of these patients present with hemodynamic instability requiring blood transfusion support $[2,3]$. Endoscopic haemostatic measures present an efficient, noninvasive method to control these kinds of serious events [2-5].

\section{Case 1}

A 71-year-old man presented at the emergency department with rectal bleeding since the past 12 hours. He had undergone TRUS-guided prostate biopsy 10
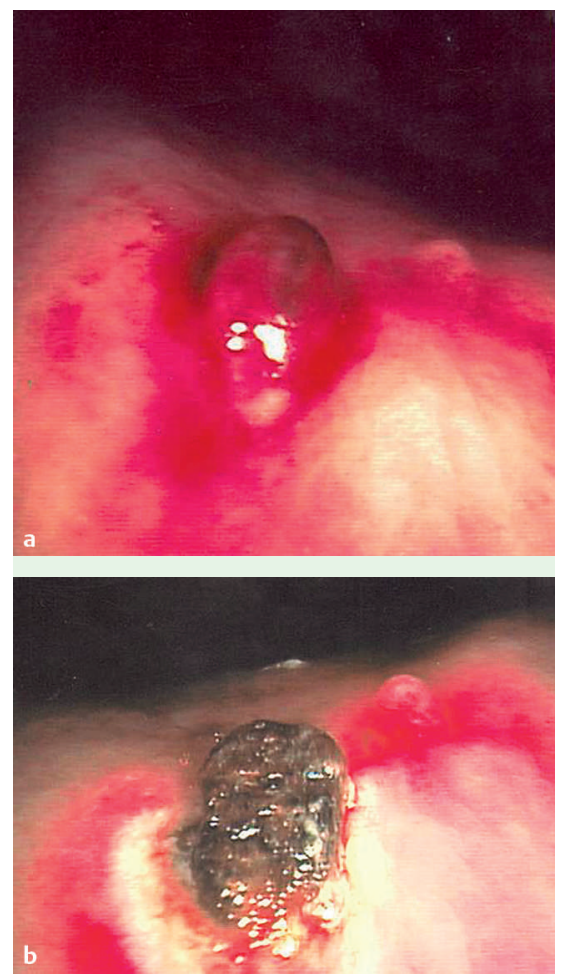

Fig. 2 a Blood vessel oozing blood near the anal verge. $\mathbf{b}$ Bleeding controlled with argon plasma coagulation (APC).

days previously and was on long-term aspirin therapy. He was pale and tachycardic. His hematocrit was $23 \%$, and platelet count and coagulation parameters were normal.

Two units of packed red blood cells were transfused. On colonoscopy, an adherent blood clot was visualized on the anterior rectal wall near the anal verge. Aspiration of the blood clot revealed a blood vessel (- Fig. 1 a).

The bleeding was stopped by administering adrenaline $10 \mathrm{~mL}(1 / 10000)$ plus $4 \mathrm{~mL}$ polidocanol (Aethoxysklerol 1\% sclerosing agent; Chemische Fabrik, Kreussler GmbH, Germany), However, the bleeding restarted 24 hours later. Elastic band ligation (EBL) was then carried out, which resolved the situation (๑ Fig. 1 b).

\section{Case 2}

$\nabla$

A 62-year-old man was admitted to the emergency department following TRUSguided prostate biopsy performed 2 hours earlier. The hemorrhage was not contained by digital compression or rectal packing. The patient was hemodynamically stable and hematologic analysis did not show a marked drop in hematocrit (42.6\% to $38.4 \%$ ). Urgent colonoscopy was carried out, which revealed active bleeding from the biopsy site in the anterior rectal wall ( $\bullet$ Fig. 2 a), which was easily controlled with argon plasma coagulation (APC) ( $\bullet$ Fig. 2 b).

In conclusion, irrespective of its accuracy, every endoscopic technique has a margin of error occasionally demanding combination therapy. In this particular context, both APC and EBL were effective.

Endoscopy_UCTN_Code_CPL_1AL_2AZ

Endoscopy_UCTN_Code_CPL_1AL_2AF

\section{B. Arroja, C. Gonçalves, F. Silva, \\ I. Cotrim, H. Vasconcelos}

Department of Gastroenterology, Hospital de Santo André EPE, Leiria, Portugal

\section{References}

1 Ghani KR, Dundas D, Patel U. Bleeding after transrectal ultrasonography-guided prostate biopsy: a study of 7-day morbidity after a six-, eight-, and twelve-core biopsy protocol. BJU Int 2004; 94: 1014 - 1020

2 Brullet E, Guevara MC, Campo R et al. Massive rectal bleeding following transrectal ultrasound-guided prostate biopsy. Endoscopy 2000; 32: $792-795$

3 Strate LL, O'Leary MP, Carr-Locke DL. Endoscopic treatment of massive rectal bleeding following prostate needle biopsy. Endoscopy $2001 ; 33$ : $981-984$

4 Geraci G, Sciumé C, Pisello F et al. Severe rectal bleeding after transrectal US-guided prostate biopsy. Case report. G Chir 2006; 27: $321-323$

5 Witte JT, Bohlman T. Endoscopic band ligation of colonic bleeding. Gastrointest Endosc 2000; 51: 117-119

\section{Bibliography}

DOI $10.1055 / \mathrm{s}-0028-1119724$

Endoscopy 2009; 41: E145

(c) Georg Thieme Verlag KG Stuttgart · New York . ISSN 0013-726X

\section{Corresponding author}

\section{B. Arroja}

Hospital de Santo André EPE, Serviço de Gastorenterologia

Rua das Olhalvas-Pousos 2410-197

Leiria, Portugal

Fax: +351-244-817087

brunoarroja@gmail.com 